Relations industrielles

Industrial Relations

\title{
Index — volume $30-1975$
}

Volume 30, numéro 4, 1975

URI : https://id.erudit.org/iderudit/028679ar

DOI : https://doi.org/10.7202/028679ar

Aller au sommaire du numéro

Éditeur(s)

Département des relations industrielles de l'Université Laval

ISSN

0034-379X (imprimé)

1703-8138 (numérique)

Découvrir la revue

Citer ce document

(1975). Index - volume 30 - 1975. Relations industrielles / Industrial Relations, 30(4), 823-843. https://doi.org/10.7202/028679ar

Tous droits réservés (C Département des relations industrielles de l'Université Laval, 1975
Ce document est protégé par la loi sur le droit d'auteur. L’utilisation des services d'Érudit (y compris la reproduction) est assujettie à sa politique d'utilisation que vous pouvez consulter en ligne.

https://apropos.erudit.org/fr/usagers/politique-dutilisation/
Cet article est diffusé et préservé par Érudit.

Érudit est un consortium interuniversitaire sans but lucratif composé de l'Université de Montréal, l'Université Laval et l'Université du Québec à

Montréal. Il a pour mission la promotion et la valorisation de la recherche.

https://www.erudit.org/fr/ 


\section{INDEX - VOL. $30-1975$ \\ Index alphabétique des articles}

Absenteeism and Health, The Effects of Job Design on Turnover, by A. Mikalachki, no 3

Academic Freedom: Arc They Compatible?, Collective Bargaining and, by $\mathrm{H}$. D. Woods, no 4

Académique: compatibilité?, Négociation collective et liberté, par H.D.

Woods, no 4

Affaires sociales, Malaise des cadres et critique des solutions proposées: application aux cadres intermédiaires des, par Laurent Bélanger, no 2

Alberta Compromise, Final Position Arbitration and Intertemporal ComCompromise: The University of, by Gene Swimmer, no 3

Alberta, Les rapports entre les secteurs public et privé en matière de salaires en, par Gene Swimmer et C.B. Williams, no 2

Alberta, The Relationship Between Public and Private Sector Wages in, by Gene Swimmer and C.B. Williams, no 2

Analysis, Marxian, by Earl F. Beach, no 4

Analysis of Factors Capable of Affecting Labour Supply: The Case of Parents of Low-Income Families, by Bertrand Belzile et Viateur Larouche, no 2

Apprentissage de la négociation collective, Les jeux dans l', par L. Tracy et R.B. Peterson, no 1

Arbitration and Intertemporal Compromise: The University of Alberta Compromise, Final Position, by Gene Swimmer, no 3

Associations d'employeurs en Israël, Structure et fonctions des, par Arie Shirom et Dan Jacobson, no 3

Associations, The Structure and Function of Israeli Employers', by Arie Shirom and Dan Jacobson, no 3

Attitudes (Les) des employeurs canadiens et américains en matière de relations professionnelles, par Mark Thompson et Larry F. Moore, no 3

Attitudes Toward Industrial Relations: A U.S.-Canadian Comparison, Managerial, by Mark Thompson and Larry F. Moore, no 3

Bargaining and Academic Freedom: Are They Compatible?, Collective, by H.D. Woods, no 4

Bargaining Any Way?, Who Wants Collective, by A.W.R. Carrothers, no 3 
Bargaining: A Review of a Report or A Tale of Two Persons, Public Sector, by William B. Cunningham, no 1

Bargaining in the Toronto Printing Industry in the Nineteenth Century, The Development of Collective, by Sally Zerker, no 1

Bargaining in University Faculties: Pros and Cons, Collective, by David M. Beatty, no 4

Bargaining in University Faculties: Pros and Cons, Collective, by Gilbert Levine, no 4

Bargaining in University Faculties: Some Cases, Collective, by Jean Boivin, no 4

Bargaining in University Faculties: Some Unresolved Dilemmas, Collective, by Donald D. Carter, no 4

$662-671$

Bargaining, Participation in Decision-Making and Collective, by Dimitri Weiss, no 4

Bargaining Rights in the Fisheries of Nova Scotia and Atlantic Canada, The Economics of, by Charles Steinberg, no 2

Bargaining Simulation: How Close to the Real Thing, Classroom $1 \mathrm{Col}$ lective, by L. Tracy and R.B. Peterson, no 1

Blue-Collar Workers, Task Specialization and Organizaitonal Commitment: An Empirical Examination Among, by Muhammad Jamal, no 4

Board, The Public Service Staff Relations, by A. Gray Gillespie, no 4 $628-641$

Cadres et critique des solutions proposées: application aux cadres intermédiaires des affaires sociales, Malaise des, par Laurent Bélanger, no 2

Cadres hiérarchiques par le Code du travail, La qualification des, par Rodrigue Blouin, no 3

$478-510$

Canada: 1957-1970, Hidden Unemployment by Age and Sex in, by Giuseppe C. Ruggeri, no 2

$181-196$

Canada: 1957-1970, Le chômage déguisé par l'âge et sexe au, par Giuseppe C. Ruggeri, no 2

$196-200$

Canada, Quelques effets des règlements sur la pratique de la médecine dans la répartition des praticiens au, par Malcolm C. Brown, no 3

$449-452$

'Canada, Some Effects of Physician Licensing Requirements on Medical Manpower Flows in Canada, Malcolm C. Brown, no 3

$436-449$

Canadian (The) Experiment with Voluntary Incomes Restraint, Degree of Labour Organization and Cyclinal Sensitivity of Employment, by William D. Walsh, no 3

Canadian (The) Industrial Strategy, by Arthur W. Donner and Fred Lazar, no 4 
Canadian Manufacturing Industries, Wage Differentials and Market Imperfections: Some Cross Section Results in, by J.C.H. Jones and L. Laudadio, no 3

Canadienne en matière de restriction volontaire des revenus, L'expérience, par William D. Walsh, no 3

$407-408$

Canadienne, les différences de salaires et les imperfections du marché: comparaisons dans l'industrie manufacturière, par J.C.H. Jones and L. Laudadio, no 3

Canadiens et américains en matière de relations professionnelles, Les attitudes des employeurs, par Mark Thompson et Larry F. Moore, no 3

Central Life Interest, On the Relationship Between Job Involvement and, by Vance F. Mitchell, Vishwanath Baba and Timothy Epps, no 2

Chômage (Le) déguisé par âge et sexe au Canada: 1957-1970, par Giuseppe C. Ruggerie, no 2

Classroom Collective Bargaining Simulation: How Close to the Real Thing, by L. Tracy and R.B. Peterson, no 1

Code du travail, La qualification des cadres hiérarchiques par le, par Rodrigue Blouin, no 3

Collective Bargaining and Academic Freedom: Are They Compatible? by H.D. Woods, no 4

Collective Bargaining Any Way?, Who Wants, by A.W.R. Carrothers, no 3

Collective Bargaining in the Toronto Printing Industry in the Nineteenth Century, The Development of, by Sally Zerker, no 1

Collective Bargaining in University Faculties: Pros and Cons, by David M. Beatty, no 4

Collective Bargaining in University Faculties: Pros and Cons, by Gilbert Levine, no 4

Collective Bargaining in University Faculties: Some Cases, by Jean Boivin, no 4

Collective Bargaining in University Faculties : Some Unresolved Dilemmas, Donald D. Carter, no 4

Collective Bargaining Simulation: How Close to the Real Thing, Classroom, by L. Tracy and R.B. Peterson, no 1

Collective dans le milieu universitaire: le pour et le contre, La négociation, par David M. Beatty, no 4

Collective dans le milieu universitaire: quelques cas, La négociation, par Jean Boivin, no 4

Collective dans le milieu universitaire: quelques dilemmes insolubles, La négociation, par Donald D. Carter, no 4 
Collective et liberté académique: compatibilité?, Négociation, par H.D. Woods, no 4

Collective, Le fondement historique de la négociation, par Sally Zerker, no 1

Collective, Les jeux dans l'apprentissage de la négociation, par L. Tracy et R.B. Peterson, no 1

'Collective?, Qui veut la convention, par A.W.R. Carrothers, no 3.

Commentaires sur la prévision des besoins en main-d'oeuvre, par $\mathbf{K}$. Weiermair, no 2

Commission ( $\mathrm{La}$ ) des relations de travail dans la fonction publique, par A. Gray Gillespie, no 4

Construction au Québec - un mythe ou une réalité?, l'insécurité d'emploi des travailleurs, par Jean Lebon, no 4

Convention collective?, Qui veut la, par A.W.R. Carrothers, no 3

Cotisation syndicale, Nature et procédure d'adoption de la, par Rodrigue Blouin, no 4

Decision-Making and Collective Bargaining, Participation in, by Dimitri Weiss, no 4

$584-585$

Demand for Manpower Training: Some Empirical Results, Determinants of the, by L.R. Truesdell, no 3

Demande de formation de la main-d'oeuvre: résultats empiriques, Facteurs déterminants de la, par L.R. Truesdell, no 3

Determinants of the Demands for Manpower Training: Some Empicical Results, by L.R. Truesdell, no 3

Developments in Saskatchewan, Teachers' Salary Differentials and the Quality of Educational Services: Recent, by P.Y. Walmsley and M. Ohtsu, no 4

Development (The) of Collective Bargaining in the Toronto Printing Industry in the Nineteenth Century, by Sally Zerker, no 1

Différences (Les) de salaires et les imperfections du marché: comparaisons dans l'industrie manufacturière canadienne, par J.C.H. Jones et L. Laudadio, no 3

Droit de négociation dans les pêcheries de l'Atlantique, Quelques aspects économiques $d u$, Charles Steinberg, no 2

Ecarts (Les) de salaire chez les enseignants et la qualité des services d'éclucation: le cas de la Saskatchewan, par P.Y. Walmsley et M. Ohtsu, no 4

Economics (The) of Bargaining Rights in the Fisheries of Nova Scotia and Atlantic Canada, Charles Steinberg, no 2 
Education: le cas de la Saskatchewan, Les écarts de salaire chez les enseignants et la qualité des services d', par P.Y. Walmsley et M. Ohtsu, no 4

Educational Services: Recent Developments in Saskatchewan, Teachers' Salary Differentials and the Quality of, by P.Y. Walmsley and M. Ohtsu, no 4

Effects (The) of Job Design on Turnover, Absenteeism and Health, by A. Mikalachki, no 3

$377-388$

Effects of Physician Licensing Requirements on Medical Manpower Flows in Canada, Some, by Malcolm C. Brown, no 3

Effets (Les) de la restructuration des postes de travail sur le roulement de la main-d'oeuvre, par A. Mikalachki, no 3

Effets des règlements sur la pratique de la médecine dans la répartition des praticiens au Canada, Quelques, par Malcolm C. Brown, no 3

$449-452$

Emploi des travailleurs de la construction au Québec - un mythe ou une réalité? L'insécurité $d$ ', par Jean Lebon, no 4

Emploi et appartenance à l'organisation, Spécialisation dans l', par $\mathrm{Mu}-$ hammad Jamal, no 4

$626-628$

Employers' Association, The Structure and Function of Israeli, by Arie Shirom and Dan Jacobson, no 3

Employeurs canadiens et américains en matière de relations professionnelles, Les attitudes des, par Mark Thompson et Larry F. Moore, no 3

Employeurs en Israël, Structure et fonctions des associations d', par Arie Shirom et Dan Jacobson, no 3

Employment, The Canadian Experiment with Voluntary Incomes Restraint Degree of Labour Organization and Cyclical Sensitivity of, by William D. Walsh, no 3

Engagement (l') au travail et l'idéal de vie, Les rapports entre, par Vance F. Mitchell, Vishwanath Baba and Timothy Epps, no 2

Enseignants et la qualité des services d'éducation: le cas de la Saskatchewan, Les écarts de salaire chez les, par P.Y. Walmsley et M. Ohtsu, no 4

Entreprise: terminologie, Le personnel dans l', par Gérard Dion, no 3

Entreprise (l') - terminologie, Les mouvements du personnel dans, par Gérard Dion, no 2

Expérience $\left(L^{\prime}\right)$ canadienne en matière de restriction volontaire des reve nus, par William D. Walsh, no 3

Facteurs affectant l'offre de travail: les personnes à faible revenu, par Bertrand Belzile et Viateur Larouche, no 2 
Facteurs déterminants de la demande de formation de la main-d'oeuvre: résultats empiriques, par L.R. Truesdell, no 3

Faculties: Pros and Cons, Collective Bargaining in University, by David M. Beatty, no 4

Faculties: Pros and Cons, Collective Bargaining in University, by Gilbert Levine, no 4

$727-736$

Faculties: Some Cases, Collective Bargaining in University, by Jean Boivin, no 4

Faculties: Some Unresolved Dilemmas, Collective Bargaining in University, by Donald D. Carter, no 4

662-671

Faible revenu, Facteurs affectant l'offre de travail: les personnes, par Bertrand Belzile et Viateur Larouche, no 2

Familles à faible revenu: tentative de mesure, Motivation au travail des, par Bertrand Belzile et Viateur Larouche, no 1

Final Position Arbitration and Intertemporal Compromise: The University of Alberta Compromise, by Gene Swimmer, no 3

533-537

Fiscal Policy, Inflation and, by André Raynauld, no 3

Fiscalité, Inflation et, par André Raynauld, no 3

Fisheries of Nova Scotia and Atlantic Canada, The Economics of Bargaining Rights in the, by Charles Steinberg, no 2

Fonction publique, La Commission des relations de travail dans la, par A. Gray Gillespie, no 4

Fonctions des associations d'employeurs en Israël, Structure et, par Arie Shirom et Dan Jacobson, no 3

$4.75-478$

Fondement (Le) historique de la négociation collective, par Sally Zerker, no 1 ,

Formation de la main-d'oeuvre: résultats empiriques, Facteurs déterminants de la demande de, par L.R. Truesdell, no 3

$4.35-436$

Formule (La) Rand, L'origine de la - Annexe, par Gérard Dion, no 4 747-772

Freedom: Are They Compatible? Collective Bargaining and Academic, by H.D. Woods, no 4

Function of Israeli Employers' Associations, The Structure and, by Arie Shirom and Dan Jacobson, no 3

Health, The Effects of Job Design on Turnover, Absenteeism an, by A. Mikalachki, no 3

Hidden Unemployment by Age and Sex in Canada: 1957-1970, by Giuseppe C. Ruggeri, no 2 
Idéal (l') de vie, Les rapports entre l'engagement au travail et, par Vance F. Mitchell, Vishwanath Baba and Timothy Epps, no 2

Incomes Restraint, Degree of Labour Organization and Cyclical Sensitivity of Employment, The Canadian Experiment with Voluntary, by William D. Walsh, no 3

Industrial Relations and Political Science, by Dimitri Weiss, no 1

Industrial Strategy, The Canadian, by Arthur W. Donner and Fred Lazar, no 4

Inflation and Fiscal Policy, by André Raynauld, no 3

Inflation et fiscalité, par André Raynauld, no 3

Insécurité ( $L$ ') d'emploi des travailleurs de la construction au Québec un mythe ou une réalité?, par Jean Lebon, no 4

Inventaire de satisfaction au travail: validation, par Viateur Larouche, no 3

Israël, Structure et fonctions des associations d'employeurs en, par Arie Shirom et Dan Jacobson, no 3

Israeli Employers' Associations, The Structure and Function of, by Arie Shirom and Dan Jacobson, no 3

Jeux (Les) dans l'apprentissage de la négociation collective, par L. Tracy and R.B. Peterson, no 1

Job Design on Turnover, Absenteeism and Health, The Effects of, by A. Mikalachki, no 3

Job Involvement and Central Life Interest, On the Relationship Between, by Vance F. Mitchell, Vishwanath Baba and Timothy Epps, no 2,

Job (The) Satisfaction Inventory: A Test of Validity, by Viateur Larouche, no 3

Labour Organization and Cyclical Sensitivity of Employment, The Canadian Experiment with Voluntary Incomes Restraint, Degree of, by William D. Walsh, no 3

Labour Relations in the Public Service: Manitoba, H.D. Woods, no 1

Labour Supply: The 'Case of Parents of Low-Incame Families, Analysis of Factors Capable of Affecting, by Bertrand Belzile et Viateur Larouche, no 2

Liberté académique: compatibilité?, Négociation collective et, par H.D. Woods, no 4

Licensing Requirements on Medical Manpower Flows in Canada, Some Effects of Physician, by Malcolm C. Brown, no 3

Line Management under the Quebec Labor Code, by Rodrigue Blouin, no 3 
Low-Income Families, Analysis of Factors Capable of Affecting Labour Supply: The Case of Parents of, by Bertrand Belzile et Viateur Larouche, no 2

Main-d'oeuvre, Commentaires sur la prévision des besoins en, par K. Weiermair, no 2 ,

Main-d'oeuvre, Les effets de la restructuration des postes de travail sur le roulement de la, par A. Mikalachki, no 3

Main-d'oeuvre: résultats enpiriques, Facteurs déterminants de la demande formation de la, par L.R. Truesdell, no 3

Malaise des cadres et critique des solutions proposées: application aux caIdres intermédiaires des affaires sociales, par Laurent Bélanger, no 2

Management Sciences - How Goes Its Philosophy, by J.W. Howard, no 2

Management under the Quebec Labor Code, Line, by Rodrigue Blouin, no 3

Managerial Attitudes Toward Industrial Relations: A U.S.-Canadian Comparison, by Mark Thompson and Larry F. Moore, no 3

Manitoba, Labour Relations in the Public Service:, by H.D. Woods, no 1

Manitoba, Les relations de travail dans le secteur public au, par H.D. Woods, no 1

Manpower Flows in Canada, Some Effects of Physician Licensing Requirements on Medical, by Malcolm C. Brown, no 3

Manpower Forecasting, A Note on, by K. Weiermair, no 2

Manpower Training: Some Empirical Results, Determinants of the Demand for, by L.R. Truesdell, no 3

Manufacturing Industries, Wage Differentials and Market Imperfections: Some Cross Section Results in Canadian, by J.C.H. Jones, no 3...

$408-422$

Marché: comparaisons dans l'industrie manufacturière canadienne, Les différences de salaires et les imperfections $d u$, par J.C.H. Jones et L. Laudadio, no 3

Market Imperfections: Some Cross Section Results in Canadian Manufacturing Industries, Wage Differentials and, by J.C.H. Jones and L. Laudadio, no 3

Marxian Analysis, by Earl F. Beach, no 4

Médecine dans la répartition des praticiens au Canada, Quelques effets des règlements sur la pratique de la, par Malcolm $\mathrm{C}$. Brown, no 3

Medical Manpower Flows in Canada, Some Effects of Physician Licensing Requirements on, by Malcolm C. Brown, no 3 
Motivation and Work: Measure, by Bertrand Belzile and Viateur Larouche, no 1

Motivation au travail des familles à faible revenu: tentative de mesure, par Bertrand Belzile et Viateur Larouche, no 1

Mouvements (Les) du personnel dans l'entreprise-terminologie, par Gérard Dion, no 2

Nature et procédure d'adoption de la cotisation syndicale, par Rodrigue Blouin, no 4

Négociation ( $\mathrm{La}$ ) collective dans le milieu universitaire: le pour et le contre, par David M. Beatty, no 4

Négociation (La) collective dans le milieu universitaire: quelques cas, par Jean Boivin, no 4

Négociation ( $L a)$ collective dans le milieu universitaire: quelques dilemnes insolubles, par Donald D. Carter, no 4

Négociation collective et liberté académique: compatibilité? par H. D. Woods, no 4

Négociation collective, Le fondement historique de la, par Sally Zerker, no 1

Négociation collective, Les jeux dans l'apprentissage de la, par L. Tracy let R.B. Peterson, no 1

Négociation collective, Participation aux décisions et, par Dimitri Weiss, no 4

$571-584$

Note $(A)$ on Manpower Forecasting, by $\mathrm{K}$. Weiermair, no 2

Nova Scotia and Atlantic Canada, The Economics of Bargaining Rights in the Fisheries of, by Charles Steinberg, no 2

On the Relationship Between Job Involvement and Central Life Interest, by Vance F. Mitchell, Vishwanath Baba and Timothy Epps, no 2

$166-177$

Organisation, Spécialisation dans l'emploi et appartenance d l', par Muhammad Jamal, no 4

$626-628$

Organisations syndicales, Les syndicats en tant qu', par Malcolm Warner, no 1

Organization and Cyclical Sensitivity of Employment, The Canadian Experiment with Voluntary Incomes Restraint, Degree of Labour, by William D. Walsh, no 3

Organizations: Strategy, Structure and the Need for Administrative Innovation, Unions as Complex, by Malcolm Warner, no 1 
Organizational Commitment: An Empirical Examination Among BlueCollar Workers, Task Specialization and, by Muhammad Jamal, no 4

Participation aux décisions et négociation collective, par Dimitri Weiss, no 8

Participation in Decision-Making and Collective Bargaining, by Dimitri Weiss, no 4

Pêcheries de l'Atlantique, Quelques aspects économiques du droit de négociation dans les, Charles Steinberg, no 2

Personnel (Le) dans l'entreprise: terminologie, par Gérard Dion, no 3

Personnel dans l'entreprise-terminologie, Les mouvements du, par Gérard Dion, no 2

Physician Licensing Requirements on Medical Manpower Flows in Canada, Some Effects of, by Malcolm C. Brown, no 3

Policy, Inflation and Fiscal, by André Raynauld, no 3

Political Science, Industrial Relations and, by Dimitri Weiss, no 1

Position Arbitration and Intertemporal Compromise: The University of Alberta Compromise, Final, by Gene Swimmer, no 3

Postes de travail sur le roulement de la main-d'oeuvre, Les effets de la restructuration des, par $\mathrm{A}$. Mikalachki, no 3

Praticiens au Canada, Quelques effets des règlements sur la pratique de la médecine dans la répartition des, par Malcolm C. Brown, no 3

Prévision des besoins en main-d'oeuvre, Commentaires sur la, par $\mathrm{K}$. Weiermair, no 2

Printing Industry in the Nineteenth Century, The Development of Collective Bargaining in the Toronto, by Sally Zerker, no 1

Prix et des salaires du Ministre Turner, Les restrictions des, par Bertrand Belzile, Pierre-Paul Proulx, no 3

Procédure d'adoption de la cotisation syndicale, Nature et, par Rodrigue Blouin, no 4

Public and Private Sector Wages in Alberta, The Relationship Between, by Gene Swimmer and C.B. Williams, no 2

Public Sector Bargaining: A Review of a Report or A Tale of Two Persons, by William B. Cunningham, no 1

Public Service: Manitoba, Labour Relations in the, by H. D. Woods, no 1

Public (The) Service Staff Relations Board, by A. Gray Gillespie, no 4

Qualification ( $\mathrm{La}$ ) des cadres hiérarchiques par le 'Code du travail, par Rodrigue Blouin, no 3 
Quebec Labor Code, Line Management under the, by Rodrigue Blouin, no 3

Québec - un mythe ou une réalité?, L'insécurité d'emploi des travailleurs de la construction au, par Jean Lebon, no 4

Quelques aspects économiques du droit de négociation dans les pêcheries de l'Atlantique, Charles Steinberg, no 2

Quelques effets des règlements sur la pratique de la médecine dans la répartition des praticiens au Canada, par Malcolm C. Brown, no 3

$449-452$

Qui veut la convention collective?, par A.W.R. Carrothers, no 3

Rand - Annexe - L'origine de la formule, par Gérard Dion, no 4

Rapport (Le) Waisberg, par Gérard Hébert, no 1

Rapports (Les) entre l'engagement au travail et l'idéal de vie, par Vance F. Mitchell, Vishwanath Baba and Timothy Epps, no 2

Rapport (Les) entre les secteurs public et privé en matière de salaires en Alberta, par Gene Swimmer et C.B. Williams, no 2

Règlements sur la pratique de la médecine dans la répartition des praticiens au Canada, Quelques effets des, par Malcolm C. Brown, no 3

$449-14152$

Relations: A U.S.-Canadian Comparison, Managerial Attitudes Toward Industrial, by Mark Thompson and Larry F. Moore, no 3

Relations de travail dans la fonction publique, La Commission des, par A. Gray Gillespie, no 4

Relations industrielles et science politique, par Dimitri Weiss, no 1

Relations in the Public Service: Manitoba, Labour, by H.D. Woods, no 1

Relations (Les) de travail dans le secteur public au Manitoba, par H.D. Woods, no 1

Relations professionnelles, Les attitudes des employeurs canadiens et américains en matière de, par Mark Thompson et Larry F. Moore, no 3

Relationship (The) Between Public and Private Sector Wages in Alberta, by Gene Swimmer and C.B. Williams, no 2

Requirements on Medical Manpower Flows in Canada, Some Effects of Physician Licensing, by Malcolm C. Brown, no 3

Restrictions (Les) des prix et des salaires du Ministre Turner, par Bertrand Belzile, Pierre-Paul Proulx, no 3

Restructuration des postes de travail sur le roulement de la main-d'oeuvre, Les effets de la, par A. Mikalachki, no 3

Revenu: tentative de mesure, Motivation au travail des familles à faible, par Bertrand Belzile et Viateur Larouche, no 1 
Revenus, L'expérience canadienne en matière de restriction volontaire des, par William D. Walsh, no 3

$407-408$

Roulement de la main-d'oeuvre, Les effets de la restructuration des postes de travail sur le, par A. Mikalachki, no 3

$388-390$

Salaire chez les enseignants et la qualité des services d'éducation le cas de la Saskatchewan, Les écarts de, par P.Y. Walmsley et M. Ohtsu, no 4

Salaires du ministre Turner, Les restrictions des prix et des, par Bertrand) Belzile, Pierre-Paul Proulx, no 3

Salaires en Alberta, Les rapports entre les secteurs public et privé en matière de, par Gene Swimmer et C.B. Williams, no 2

Salaires et les imperfections du marché: comparaisons dans l'industrie manufacturière canadienne, Les différence de, par J.C.H. Jones et L. Laudadio, no 3

Salary Differentials and the Quality of Educational Services: Recent Developments in Saskatchewan, Teachers', by P.Y. Walmsley and M. Ohtsu, no 4

Saskatchewan, Les écarts de salaire chez les enseignants et la qualité des services d'éducation: le cas de la, par P.Y. Walmsley et M. Ohtsu, no 4

Saskatchewan, Teachers' Salary Differentials and the Quality of Educational Services: Recent developments in, by P.Y. Walmsley and M. Ohstu, no 4

Satisfaction au travail: validation, Inventaire de, par Viateur Larouche, no 3

Satisfaction Inventory: A Test of Validity, The Job, by Viateur Larouche, no 3

Science politique, Relations industrielles et, par Dimätri Weiss, no 1 30- 41

Secteur public au Manitoba, Les relations de travail dans le, par H.D. Woods, no 1

Some Effects of Physician Licensing Requirements on Medical Manpower Flows in Canada, by Malcolm C. Brown, no 3

Spécialisation dans l'emploi et appartenance à l'organisation, par Muhammad Jamal, no 4

Specialization and Organizational Commitment: An Empirical Examination Among Blue-Collar Workers, Task, by Muhammad Jamal, no 4 
Strategy, The Canadian Industrial, by Arthur W. Donner and Fred Lazar, no 4

Structure (The) and Function of Israeli Employers' Associations, by Arie Shirom and Dan Jacobson, no 3

Structure and the Need for Administrative Innovation, Unions as Complex Organizations: Strategy, by Malcolm Warner, no 1

Structure et fonctions des associations d'employeurs en Israël, par Arie Shirom et Dan Jacobson, no 3

Syndicale, Nature et procédure d'adoption de la cotisation, par Rodrigue Blouin, no 4

Syndicales, Les syndicats en tant qu'organisations, par Malcolm Warner, no 1

Syndicats (Les) en tant qu'organisations syndicales, par Malcolm Warner, no 1

Task Specialization and Organizational Commitment: An Empirical Examination Among Blue-Collar Workers, by Muhammad Jamal, no 4

Teachers' Salary Differentials and the Quality of Educational Services: Recent Developments in Saskatchewan, by P. Y. Walmsley and M. Ohtsu, no 4

Terminologie, Le personnel dans l'entreprise:, par Gérard Dion, no 3

Terminologie, Les mouvements du personnel dans l'entreprise -, par Gérard Dion, no 2

Toronto Printing Industry in the Nineteenth Century, The Development of Collective Bargaining in the, by Sally Zerker, no 1

Training: Some Empirical Results, Determinants of the Demand for Manpower, by L.R. Truesdell, no 3

Travail dans le secteur public au Manitoba, Les relations de, par H.D. Woods, no 1

Travail des familles à faible revenu: tentative de mesure, Motivation au, par Bertrand Belzile et Viateur Larouche, no 1

Travail sur le roulement de la main-d'oeuvre, Les effets de la restructuration des postes de, par $\mathrm{A}$. Mikalachki, no 3

Travail: validation, Inventaire de satisfaction au, par Viateur Larouche, no 3

Travailleurs de la construction au Québec - un mythe ou une réalité? L'insécurité d'emploi des, par Jean Lebon, no 4

Turnover, Absenteeism and Health, The Effects of Job Design on, by A. Mikalachki, no 3 
Unemployment by Age and Sex in Canada: 1957-1970, Hidden, by Giuseppe C. Ruggeri, no 2

Unions as Complex Organizations: Strategy, Structure and the Need for Administrative Innovation, by Malcolm Warner, no 1 .

Universitaire: le pour et le contre, La négociation collective dans le milieu, par David M. Beatty, no 4

Universitaire: quelques cas, La négociation collective dans le milieu, par Jean Boivin, no 4

Universiaire: quelques dilemmes insolubles, La négociation collective dans le milieu, par Donald D. Carter, no 4

University Faculties: Pros and Cons, Collective Bargaining in, by David M. Beatty, no 4

University Faculties: Pros and Cons, Collective Bargaining in by Gilbert Levine, no 4

University Faculties: Some Cases, Collective Bargaining in, by Jean Boivin, no 4

University Faculties: Some Unresolved Dilemmas, 'Collective Bargaining in, by Donald D. Carter, no 4

U.S.-Canadian Comparison, Managerial Attitudes Toward Industrial Relations:, by Mark Thompson and Larry F. Moore, no 3

Waisberg, Le rapport, par Gérard Hébert, no 1

Wage Differentials and Market Imperfections: Some Cross Section Results in Canadian Manufacturing Industries, by J.C.H. Jones and L. Laudadio, no 3

Wages in Alberta, The Relationship Between Public and Private Sector, by Gene Swimmer and C.B. Williams, no 2

Who Wants Collective Bargaining Any Way?, by A.W.R. Carrothers, no 3

Work: Measure, Motivation and, by Bertrand Belzile and Viateur Larouche, no 1

Workers, Task Specialization and Organizational Commitment: An Empirical Examination Among Blue-Collar, by Muhammad Jamal, no 4 


\section{Auteurs des ouvrages recensés}

BARNES, L.W.C.S., Consult and Advise: A History of the National Joint Council of the Public Service of Canada 1944-1974, (A. Gray Gillespie), no 4

BERCUSON, David Jay, Confrontation at Winnipeg: Labour, Industrial Relations and the General Strike, (Gary N. Chaison), no 1

$129-130$

BOLINO, August C., Career Education, (Jean Sexton), no 2

283-284

BUREAU INTERNATIONAL DU TRAVAIL, Les autorités publiques et le droit à la protection des fonds et autres biens syndicaux, (Rodrigue Blouin), no 1

BUREAU INTERNATIONAL DU TRAVAIL, Les entreprises multinationales et la politique socíale, (Gérard Dion), no 1

BUREAU INTERNATIONAL DU TRAVAIL, Recommandations internationales sur les statistiques du travail, (Gérard Dion), no 4 .

CANADIAN COUNCIL ON SOCIAL DEVELOPMENT, Guaranteed Annual Income: An Integrated Approach, (Douglas A. Smith), no 2

CAPARROS, E. et J. GOULET, La documentation juridique, Références et abréviations, (Rodrigue Blouin), no 1

CONSEIL ÉCONOMIQUE DU CANADA, Au-delà des frontières, (Carmine Nappi), no 4

CONSEIL ÉCONOMIQUE DU CANADA, Objectifs économiques et indicateurs sociaux, (Claude Masson), no 1

CONSEIL ÉCONOMIQUE DU CANADA, Pour une croissance plus stable de la construction, (Bertrand Belzile), no 1

CRANE, David P., Personnel Management, A Situational Approach, (Jean Ladouceur), no 4

DESCÔTEAUX, J.G., Répertoire de décisions en application du Code du travail, tome II, Mesures disciplinaires, (Rodrigue Blouin), no 4

DICHTER, E., The Naked Manager, (Alain Larocque) no 3

$800-801$

DUNLOP, John T., Frederick H. HARBISON, Clark KERR and Charles A. MYERS, Industrialialism and Industrial Man Reconsidered, (Jean Boivin), no 4

DUSSAULT, René, Traité de droit administratif canadien et québécois, (Rodrigue Blouin), no 2

GHESTIN, J., Sécurité sociale, (Jean Sexton), no 2

HOLLAND, John W. and Michael L. SKOLNIK, Public Policy and Manpower Development, (Jean Sexton), no 4 
HUMBLE, John W., How to Manage by Objectives, (Laurent Bélanger), no 1

HURTUBISE, Rolland, Les systèmes d'information aux fins de gestion, (Jean Ladouceur) no 2

JAIN, H.C., Contemporary Issues in Canadian Personnel Administration, (Roland D. Thériault), no 2

JOHNSON, Miriam, Counter Point: The Changing Employment Service, (Jean Sexton), no 2

KUMAR, Pradeep, Relative Wage Differentials in Canadian Industries (Paul Phillips), no 4

808-809

LAZARUS, Morden, Years of Hard Labour, (Gérard Dion), no 2

LEESON, R.A., Strike: A Live History, 1887-1971, (Jean Boivin), no 2

LEFEBVRE, Gérald, Le management d'aujourd'hui, Savoir organiser, Savoir décider, (Jean-Pierre Beaulieu), no 4

LEMAY, Denis, Méthode de recherche en droit québécois et canadien, (Rodrigue Blouin), no 1

LEVITAN, Sar A. and Robert TAGGART III, Employment and Earning Inadequacy: A New Social Indicator, (Claude Rondeau), no 2

MANGUM, Garth L. and David SNEDEKER, Manpower Planning for Local Labor Markets, (Jean Sexton), no 3

MANGUM, Garth L. and John WALSH, A Decade of Manpower Training and Development, (Jean Sexton), no 1

MIERNYK, William H., The Economics of Labor and Collective Bargaining, (Jean Sexton), no 3

MINER, John B. and Mary G. MINER, Personnel and Industrial Relations: A Managerial Approach, (Laurent Bélanger), no 3

MORIN, Pierre, Le travail dans l'entreprise et la société moderne, (Gilles Dussault), no 3

MORRIS, Richard B., Labor and Management, the Great Contemporary Issues, (Jean Boivin), no 3

MURRAY, John F. and Thomas J. VON DER EMBSE, Organizational Behavior: Critical Incidents and Analysis, (Laurent Bélanger), no 2

PAGÉ, Dominique, Petit dictionnaire de droit québécois et canadien (Gérard Dion), no 4

REDDIN, W.J. and R. STUART-KOTZE, Effective Situational Diagnosis: A Programmed Learning Text Based on the 3-D Theory, (Laurent Bélanger), no 1

SAINSAULIEU, Renaud, Les relations de travail à l'usine, (Laurent Bélanger), no 3 
STIEBER, Jack, Public Employee Unionism: Structure, Growth, Policy, (Jean Boivin), no 2

SWERTZ, Paul, Éléments de sociologie hospitalière, (Gilles Dussault), no 1

$128-129$

THE LABOUR RELATIONS LAW CASEBOOK GROUP, Labour Relations Law, Cases-Materials and Commentary, (Rodrigue Blouin), no 1

YANEY, Joseph P., Personnel Management, Reaching Organizational and Human Goals (Jean Ladouceur), no 3

YODER, Dale and Herbert G. HENEMAN, Motivation and Commitment, (Jean-Pierre Beaulieu), no 3

\section{Auteurs d'articles}

BEACH, Earl F., Marxian Analysis, no 4

BEATTY, David M., Collective Bargaining in University Faculties - Pros and Cons, no 4

BEATTY, David., La négociation collective dans le milieu universitaire le pour et le contre, no 4

BÉLANGER, Laurent, Malaise des cadres et critique des solutions proposées: application aux cadres intermédiaires des affaires sociales, no 2

BELZILE, Bertrand, Les restrictions des prix et des salaires du Ministre Turner, no 3

BELZILE, Bertrand and Viateur LAROUCHE, Analysis of Factors Capable of Affecting Labour Supply: The Case of Parents of LowIncome Families, no 2

BELZILE, Bertrand et Viateur LAROUCHE, Facteurs affectant l'offre de travail: les personnes à faible revenu, no 2

BELZILE, Bertrand and Viateur LAROUCHE, Motivation and Work: Measure), no 1 ,

BELZILE, Bertrand et Viateur LAROUCHE, Motivation au travail des familles à faible revenu: tentative de mesure, no 1

BLOUIIN, Rodrigue, La qualification des cadres hiérarchiques par le Code du travail, no 3

BLOUIN, Rodrigue, Line Management under the Quebec Labor Code, no 3 
BLOUIN, Rodrigue, Nature et procédure d'adoption de la cotisation syndicale, no 4

BOIVIN, Jean, Collective Bargaining in University Faculties - Some Cases, no 4

BOIVIN, Jean, La négociation collective dans le milieu universitaire Quelques cas, no 4

BROWN, Malcolm C., Quelques effets des règlements sur la pratique de la médecine dans la répartition des practiciens au Canada, no 3,

BROWN, Malcolm C., Some Effects of Physician Licensing Requirements on Medical Manpower Flows in Canada, no 3,

CARROTHERS, A.W.R., Qui veut la convention collective?, no 3

CARROTHERS, A.W.R., Who Wants Collective Bargaining Any Way?, no 3

CARTER, Donald D., Collective Bargaining in University Faculties Some Unresolved Dilemmas, no 4

CARTER, Donald D., La négociation collective dans le milieu universitaire - Quelques dilemmes insolubles, no 4

CUNNINGHAM, William B., Public Sector Bargaining: A Review of $A$ Report or A Tale of Two Persons, no 1

DION, Gérard, L'origine de la formule Rand - Annexe -

DION, Gérard, Le personnel dans l'entreprise: terminologie, no 3

DION, Gérard Les mouvements du personnel dans l'entreprise - terminologie, no 2

DONNER, Arthur W. and Fred LAZAR, The Canadian Industrial Strategy, no 4

$775-782$

GILlESPIE, A. Gray, La Commission des relations de travail dans la fonction publique, no 4

GILlESPIE, A. Gray, The Public Service Staff Relations Board, no 4 HÉBERT, Gérard, Le rapport Waisberg, no 1

HOWARD, J. W., Management Science - How Goes Its Philosophy, no 2

JAMAL, Muhammad, Spécialisation dans l'emploi et appartenance à l'organisation, no 4

JAMAL, Muhammad, Task Specialization and Organizational Commitment: An Empirical Examination Among Blue-Collar Workers, no 4

JONES, J.C.H. and L. LAUDADIO, Les différences de salaires et les imperfections du marché: comparaisons dans l'industrie manufacturière canadienne, no 3 
JONES, J.C.H. and L. LAUDADIO, Wage Differentials and Market Imperfections: Some Cross Section Results in Canadian Manufacturing Industries, no 3

$408-422$

LAROUCHE, Viateur, Inventaire de satisfaction au travail: validation, no 3

LAROUCHE, Viateur, The Job Satisfaction Inventory: $A$ Test of Validity, no 3

LEBON, Jean, L'insécurité d'emploi des travailleurs de la construction au Québec - un mythe ou une réalité?, no 4

736-747

LEVINE, Gilbert, Collective Bargaining in University Faculties - Pros and Cons, no 4

MIKALACHKI, A., Les effets de la restructuration des postes de travail sur le roulement de la main-d'oeuvre, no 3

$388-390$

MIKALACHKI, A., The Effects of Job Design on Turnover, Absenteeism and Health, no 3

$377-388$

MITCHELLL, Vance F., Vishwanath BABA et Timothy EPPS, Les rapports entre l'engagement au travail et l'idéal de vie, no 2

$177-181$

MITCHELL, Vance F., Vishwanath BABA and Timothy EPPS, On the Relationship Between Job Involvement and Central Life Interest, no 2

PROULX, Pierre-Paul, Les restrictions des prix et des salaires du Ministre Turner, no 3

RAYNAULD, André, Inflation and Fiscal Policy, no 3

RAYNAULD, André, Inflation et fiscalité, no 3

RUGGERI, Giuseppe C., Hidden Unemployment by Age and Sex in Canada, 1957-1970, no 2

RUGGERI, Giuseppe C., Le chômage déguisé par âge et sexe au Canada, $1957-1970$, no 2

SHIROM, Arie et Dan JACOBSON, Structure et fonctions des associations d'employeurs en Israël, no 3

SHIROM, Arie and Dan JACOBSON, The Structure and Function of Israeli Employers' Associations, no 3

SWIMMER, Gene, Final Position Arbitration and Intertemporal Compromise: The University of Alberta Compromise, no 3

SWIMMER, Gene et C.B. WILLIAMS, Les rapports entre les secteurs public et privé en matière de salaires en Alberta, no 2

SWIMMER, Gene and C.B. WILLIAMS, The Relationship Between Public and Private Sector Wages in Alberta, no 2 
STEINBERG, Charles, Quelques aspects économiques du droit de négociation dans les pêcheries de l'Atlantique, no 2

STEINBERG, Charles, The Economics of Bargaining Rights in the Fisheries of Nova Scotia and Atlantic Canada, no 2

THOMPSON, Mark et Larry F. MOORE, Les attitudes des employeurs canadiens et américains en matière de relations professionnelles, no 3

THOMPSON, Mark and Larry F. MOORE, Managerial Attitudes Toward Industrial Relations: A U.S.-Canadian Comparison, no 3

TRACY, L. and R.B. PETERSON, Classroom Collective Bargaining Simulation: How Close to the Real Thing, no 1

TRACY, L. et R.B. PETERSON, Les jeux de l'apprentissage de la négociation collective, no 1

TRUESDELL, L.R., Determinants of the Demand for Manpower Training: Some Empirical Results, no 3

TRUESDELL, L.R., Facteurs déterminants de la demande de formation de la main-d'oeuvre: résultats empiriques, no 3

WALMSLEY, P.Y. et M. OHTSU, Les écarts de salaire chez les enseignants et la qualité des services d'éducation: le cas de la Saskatchewan, no 4

$610-612$

WALMSLEY, P.Y. and M. OHTSU, Teachers' Salary Differentials and the Quality of Educational Services: Recent Developments in Saskatchewan, no 4

WALSH, William D., L'expérience canadienne en matière de restriction volontaire des revenus, no 3

WALSH, William D., The Canadian Experiment with Voluntary Incomes Restraint, Degree of Labour Organization and Cyclical Sensitivity of Employment, no 3

WARNER, Malcolm, Les syndicats en tant qu'organisations complexes, no 1

WARNER, Malcolm, Unions as Complex Organizations: Strategy, Structure and the Need for Administrative Innovation, no 1

WEIERMAIR, K., A Note on Manpower Forecasting, no 2

WEIERMAIR, K., Commentaires sur la prévision des besoins en maind'oeuvre, no 2

WEISS, Dimitri, Industrial Relations and Political Science, no 1

WEISS, Dimitri, Participation aux décisions et négociation collective, no 4 
WEISS. Dimitri, Participation in Decision-Making and Collective Bargaining, no 4 $584-585$

WEISS, Dimitri, Relations industrielles et sciences politique, no 1 $30-31$

WOODS, H.D., Collective Bargaining and Academic Freedom: Are They Compatible?, no 4

WOODS, H.D., Labour Relations in the Public Service: Manitoba, no 1 $3-27$

WOODS, H.D., Les relations de travail dans le secteur public au Manitoba, no 1

WOODS, H.D., Négociation collective et liberté académique: compatibilité?, no 4

ZERKER, Sally, Le fondement historique de la négociation collective, no 1

ZERKER, Sally, The Development of Collective Bargaining in the Toronto Printing Industry in the Nineteenth Century, no 1 